117:1893-1901. doi:10.1172/JCI31721.

8. Jackson, E.L., et al. 2001. Analysis of lung tumor initiation and progression using conditional expression of oncogenic K-ras. Genes Dev. 15:3243-3248.

9. Kemp, C.J., Donehower, L.A., Bradley, A., and Balmain, A. 1993. Reduction of p53 gene dosage does not increase initiation or promotion but enhances malignant progression of chemically induced skin tumors. Cell. 74:813-822.

10. Dittmer, D., et al. 1993. Gain of function mutations in p53. Nat. Genet. 4:42-46.

11. Coussens, L.M., and Werb, Z. 2002. Inflammation and cancer. Nature. 420:860-867.

12. Marjolin, J.N. 1828. Ulcère [In French]. In Dictionnaire de médecine. Paris Béchet jeune. Paris, France. 31-50.

13. Mueller, M.M. 2006. Inflammation in epithelial skin tumours: old stories and new ideas. Eur. J. Cancer. 42:735-744.

14. Moore, R.J., et al. 1999. Mice deficient in tumor necrosis factor-alpha are resistant to skin carcinogenesis. Nat. Med. 5:828-831.

15. Coussens, L.M., Tinkle, C.L., Hanahan, D., and Werb, Z. 2000. MMP-9 supplied by bone marrowderived cells contributes to skin carcinogenesis. Cell. 103:481-490.

16. Girardi, M., et al. 2001. Regulation of cutaneous malignancy by gammadelta $\mathrm{T}$ cells. Science. 294:605-609.
17. Nibbs, R.J.B., et al. 2007. The atypical chemokine receptor D6 suppresses the development of chemically induced skin tumors. J. Clin. Invest. 117:1884-1892. doi:10.1172/JCI30068.

18. Jamieson, T., et al. 2005. The chemokine receptor D6 limits the inflammatory response in vivo. Nat. Immunol. 6:403-411.

19. Berhane, T., Halliday, G.M., Cooke, B., and Barnetson, R.S. 2002. Inflammation is associated with progression of actinic keratoses to squamous cell carcinomas in humans. Br. J. Dermatol. 146:810-815.

20. Owens, D.M., Wei, S.-J.C., and Smart, R.C. 1999. A multihit, multistage model of chemical carcinogenesis. Carcinogenesis. 20:1837-1844.

\title{
Hepcidin regulation: ironing out the details
}

\author{
Ivana De Domenico, Diane M. Ward, and Jerry Kaplan
}

Department of Pathology, University of Utah School of Medicine, Salt Lake City, Utah, USA.

\begin{abstract}
Hepcidin is a peptide hormone secreted by the liver that plays a central role in the regulation of iron homeostasis. Increased hepcidin levels result in anemia while decreased expression is the causative feature in most primary iron overload diseases. Mutations in hemochromatosis type 2 (HFE2), which encodes the protein hemojuvelin (HJV), result in the absence of hepcidin and an early-onset form of iron overload disease. HJV is a bone morphogenetic protein (BMP) coreceptor and HJV mutants have impaired BMP signaling. In this issue of the JCI, Babitt and colleagues show that BMPs are autocrine hormones that induce hepcidin expression (see the related article beginning on page 1933). Administration of a recombinant, soluble form of $\mathrm{HJV}$ decreased hepcidin expression and increased serum iron levels by mobilizing iron from splenic stores. These results demonstrate that recombinant $\mathrm{HJV}$ may be a useful therapeutic agent for treatment of the anemia of chronic disease, a disorder resulting from high levels of hepcidin expression.
\end{abstract}

\section{Regulation of iron homeostasis}

In all species, the concentration of iron in biological fluids is tightly regulated to provide iron as needed and to avoid toxicity, because iron excess can lead to the generation of reactive oxygen species. Iron homeostasis in mammals is regulated at the level of intestinal absorption, as there is no excretory pathway for iron. Hepcidin, a circulating peptide hormone, is the master regulator of systemic iron homeostasis, coordinating the use and storage of iron with iron acquisition (1). This hormone is primarily produced by hepatocytes and is a negative regulator of iron entry into plasma (Figure 1). Hepcidin acts by binding to ferroportin, an iron

Nonstandard abbreviations used: BMP, bone morphogenetic protein; HJV, hemojuvelin.

Conflict of interest: The authors have declared that no conflict of interest exists.

Citation for this article: J. Clin. Invest. 117:1755-1758 (2007). doi:10.1172/JCI32701. transporter present on cells of the intestinal duodenum, macrophages, and cells of the placenta. Binding of hepcidin induces ferroportin internalization and degradation (2). The loss of ferroportin from the cell surface prevents iron entry into plasma (Figure 1A). Decreased iron entry into plasma results in low transferrin saturation, and less iron is delivered to the developing erythroblast. Conversely, decreased expression of hepcidin leads to increased cell surface ferroportin and increased iron absorption (Figure 1C).

Plasma hepcidin levels are regulated by different stimuli, including cytokines, plasma iron, anemia, and hypoxia. Dysregulation of hepcidin expression results in iron disorders. Overexpression of hepcidin leads to the anemia of chronic disease, while low hepcidin production results in hereditary hemochromatosis with consequent iron accumulation in vital organs (Figure 1). Most hereditary iron disorders result from inadequate hepcidin pro- duction relative to the degree of tissue iron accumulation. Impaired hepcidin expression has been shown to result from mutations in any of 4 different genes: transferrin receptor 2 (TFR2), hemochromatosis (HFE), hemochromatosis type 2 (HFE2), and hepcidin antimicrobial peptide (HAMP). Mutations in HAMP, the gene that encodes hepcidin, result in iron overload disease, as the absence of hepcidin permits constitutively high iron absorption. The role for other genes (TFR2, HFE, and HFE2) in the regulation of hepcidin production has been unclear. A study presented in this issue of the JCI by Babitt et al. (3) provides a mechanistic understanding of the role of hemojuvelin (HJV; encoded by HFE2) in the regulation of hepcidin production.

\section{Regulation of hepcidin expression}

Regulation of hepcidin expression appears to occur at the level of transcription (Figure 2). Inflammatory cytokines, predominately IL-6, induce transcription of HAMP in hepatocytes. This induction involves the activation of Stat3 (3) and binding of Stat 3 to a regulatory element in the HAMP promoter (4-6).

A second mode of hepcidin regulation depends upon signaling through the bone morphogenetic protein/Smad (BMP/Smad) pathway. BMPs are cytokines in the TGF- $\beta$ family. These ligands play key roles in regulating cell proliferation, differentiation, apoptosis, and migration in tissues. In particular, BMPs play essential roles in cardiac, neural, and cartilaginous differentiation. All BMPs share a common signaling pathway, which involves 


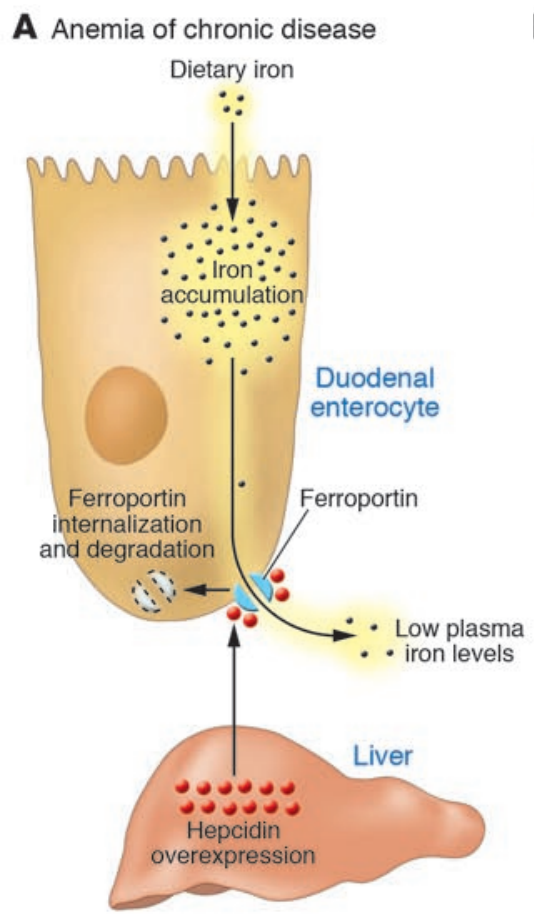

B Normal

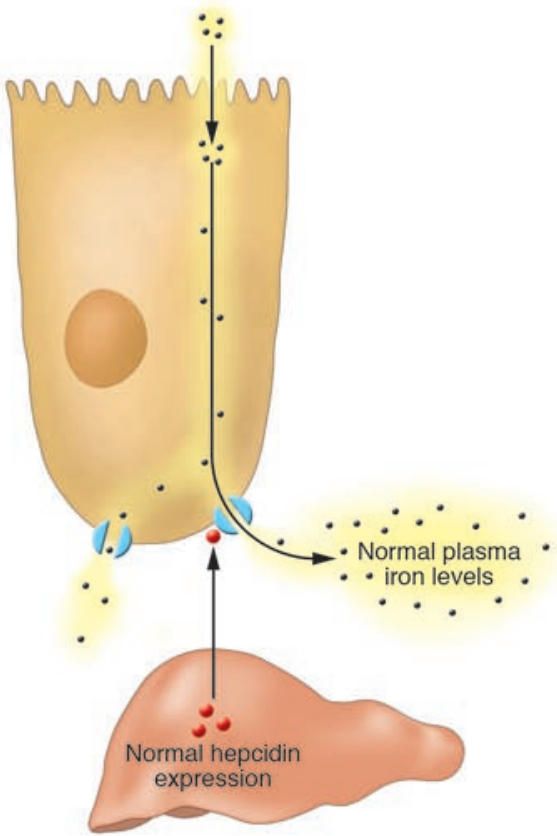

C Hemochromatosis (iron overload)

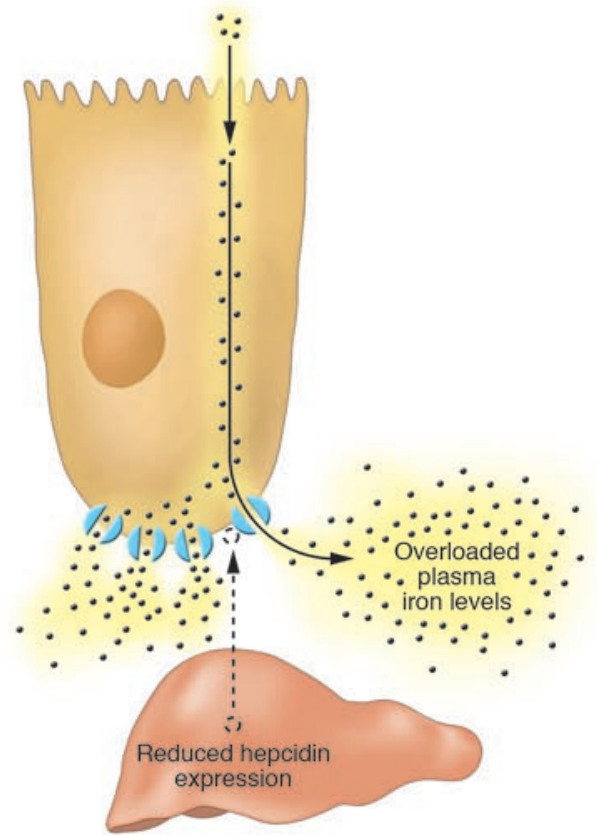

Figure 1

Hepcidin-mediated regulation of iron homeostasis. (A) Increased hepcidin expression by the liver results from inflammatory stimuli. High levels of hepcidin in the bloodstream result in the internalization and degradation of the iron exporter ferroportin. Loss of cell surface ferroportin results in macrophage iron loading, low plasma iron levels, and decreased erythropoiesis due to decreased transferrin-bound iron. The decreased erythropoiesis gives rise to the anemia of chronic disease. (B) Normal hepcidin levels, in response to iron demand, regulate the level of iron import into plasma, normal transferrin saturation, and normal levels of erythropoiesis. (C) Hemochromatosis, or iron overload, results from insufficient hepcidin levels, causing increased iron import into plasma, high transferrin saturation, and excess iron deposition in the liver.

binding of BMP to type I and type II cell serine/threonine kinase receptors, forming a BMP-type I/type II receptor complex. This complex results in the phosphorylation of an intracellular protein termed RSmad. The specificity of BMP ligand responses is due to the multiplicity of more than 20 BMPs, 7 type I receptors, 5 type II receptors, and 5 RSmads (7). The RSmads, however, combine with a single, common Smad family member, Smad4 (also referred to as Co-Smad), and that complex translocates into the nucleus and activates transcription of target genes (Figure 2). Wang et al. demonstrated that deletion of $S$ mad4 results in embryonic lethality but that liver-specific inactivation of Smad4 results in loss of hepcidin synthesis and an iron overload phenotype similar to the phenotype seen in hepcidin-knockout mice (8). Babitt et al. also showed that mice with a deletion in the Smad4 gene were unable to synthesize hepcidin in response to inflammatory stimuli or to iron load (3). This result was the first to our knowledge to show that the BMP/Smad4 pathway is critical to hepcidin expression.

\section{BMP signaling by HJV regulates hepcidin expression}

Babitt and colleagues previously demonstrated that HJV acts as a BMP coreceptor in vitro, which facilitates the activation of the BMP-type I/type II receptor complex (9). Mutations in HFE2, the gene that encodes HJV, lead to early-onset iron overload disease (10). This form of juvenile hemochromatosis is typified by the absence of hepcidin and leads to heart disease, liver iron overload, and diabetes and is indistinguishable from the effects of HAMP mutations in patients. HJV is a glycosylphosphatidyl inositol-linked cell surface protein expressed predominantly in skeletal muscle and hepatocytes. The most informative structural feature of HJV is that it has homology to a small family of proteins involved in neural guidance. Members of this family (e.g., RBMCA, RGMCB, and DRAGON) bind BMPs and enhance BMP-mediated signal- ing (10). These previous results suggested that HJV's ability to function as a BMP coreceptor was required for HJV to alter hepcidin expression and affect systemic iron homeostasis.

\section{In vivo effect of BMP and HJV}

In the present study, Babitt and colleagues extend their observations on the relationship between HJV and BMPs in hepcidin expression (3). First, they demonstrate that BMP receptor expression is selective for specific members of the TGF- $\beta$ superfamily and is not activated by TGF- $\beta$, activins, or all BMPs. Second, they show in vitro that hepatocytes secrete BMPs and that siRNA silencing of specific BMPs reduces hepcidin expression. Thus, BMPs are autocrine regulators. Third, they show in vivo that injection of BMPs into wildtype mice increases hepcidin expression and concomitantly decreases serum iron levels. Fourth, they demonstrate that a recombinant, soluble form of HJV binds BMPs and acts as a BMP antagonist, decreasing hepcidin transcription. The 


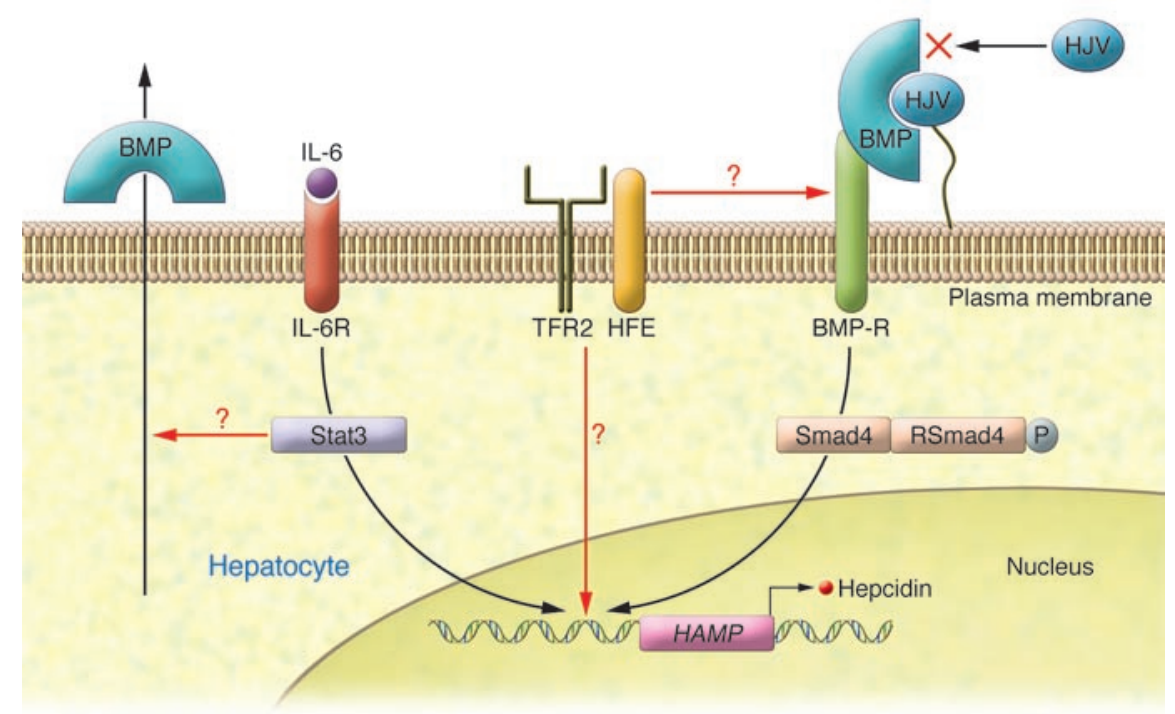

Figure 2

Transcriptional regulation of hepcidin by the BMP/Smad pathway. Hepcidin transcription depends upon signaling through BMP receptors (BMP-Rs) and downstream Smads. BMPs can act as autocrine or paracrine hormones. Binding of BMP to cell surface HJV positions BMP to activate BMP receptors. Activation of BMP receptors leads to the generation of phosphorylated RSmads, which dimerize with Smad4. The $\mathrm{RSmad} / \mathrm{Smad} 4$ heterodimer translocates into the nucleus and activates transcription of the HAMP gene, which encodes hepcidin. Soluble HJV binding to BMP prevents the formation of a cell surface BMP-HJV complex and blocks activation of BMP receptors. Inflammatory cytokines such as IL-6 bind to IL-6 receptors (IL-6Rs), activating Stat3, which also binds to the HAMP promoter. Stat3 activation requires the presence of Smad4, as deletion of the Smad4 gene prevents IL-6 induction of hepcidin. Smad4 is downstream of TFR2 and HFE, which suggests that the signal provided by these proteins also activates the HAMP promoter or that these membrane proteins affect BMP receptor signal transmission. In their study in this issue of the $\mathrm{JCl}$, Babitt and colleagues demonstrate in vivo that soluble HJV binds BMPs produced by the liver, leading to alteration in iron homeostasis (3).

observation that soluble BMPs decrease hepcidin expression in hepatocytes in vitro was shown previously (11). To our knowledge, Babitt et al. (3) are the first to show that soluble HJV inhibits hepcidin expression in vivo. Injection of soluble HJV for a period of weeks resulted in increased serum iron and increased liver iron. Soluble HJV reduced hepcidin transcripts in liver, decreasing the amount of phosphorylated RSmad. This observation provides proof that the effect of soluble $\mathrm{HJV}$ is to reduce BMP signaling events. The authors also show that soluble HJV reduces hepcidin expression in response to IL-6 (3). The observation that soluble HJV administration leads to increased liver and plasma iron but decreased hepcidin expression indicates that BMP signaling can modify hepcidin expression in response to other stimuli. These results are aligned with the observations of Wang et al. that neither iron nor IL- 6 can induce hepcidin expression in mice with a liverspecific Smad4 gene deletion (8).

There are several reasons why these results are important. First, they suggest the possibility of pharmacological inter- vention for disorders resulting from excessive hepcidin production, as seen in the anemia of chronic disease. Second, they provide a mechanistic explanation for the role of HJV in iron overload disease. Third, the observation that soluble HJV affects hepcidin production provides an explanation for what was initially, to us, a curious observation that the highest expression of HJV is found in skeletal muscle. The recent finding that HJV is released from skeletal muscle in iron deficiency suggests that this tissue may play a critical role in iron homeostasis (12).

BMPs are critical for expression of hepcidin, which leads to the question: Is expression of BMPs regulated in hepatocytes? The present study (3) and that previously reported by Wang et al. (8) provide compelling data that the BMP signaling pathway is critical for IL-6 induction, prompting us to ask: How do Stat 3 and Smad signaling interact? These pathways might interact at the level of the HAMP/ hepcidin promoter, but an interesting possibility is that activated Stat 3 might regulate hepatocyte BMP expression, as activated Stat 3 in other tissues induces the expression of BMPs (13). Finally, how do HFE and TFR2 interact with BMPs and the Smad signaling system? Addition of BMPs to hepatocytes cultured from mice with targeted deletions in the HFE or TFR2 genes showed induction of hepcidin to levels that were similar to those in wildtype hepatocytes (14). This observation, together with the finding that Smad4 signaling is required for hepcidin expression regardless of stimuli, suggests that HFE and TFR2 might work upstream of BMP. A recent study has shown that HFE and TFR2 interact and that this interaction may provide a downstream signal that affects hepcidin expression (15). Does the HFE/TFR2 complex interact with the cell surface BMP/HJV/BMP receptor complex, or do intracellular signals provided by the HFE/TFR2 complex act on the HAMP/ hepcidin promoter? The finding that the $\mathrm{BMP} /$ Smad system is fundamental to hepcidin expression provides a valuable starting point to deciphering these issues.

\section{Acknowledgments}

The research work in the laboratory of J. Kaplan is supported by NIH grants 
DK070947 and DK30534. The authors express their appreciation to Richard Ajioka for assistance with Figure 2.

Address correspondence to: Jerry Kaplan, Department of Pathology, University of Utah School of Medicine, 50 North Medical Drive, Salt Lake City, Utah 84106, USA. Phone: (801) 581-7427; Fax: (801) 581-4517; E-mail: jerry.kaplan@path.utah.edu.

1. Nemeth, E., and Ganz, T. 2006. Regulation of iron metabolism by hepcidin. Annu. Rev. Nutr. 26:323-342.

2. Nemeth, E., et al. 2004. Hepcidin regulates cellular iron efflux by binding to ferroportin and inducing its internalization. Science. 306:2090-2093.

3. Babitt, J.L., et al. 2007. Modulation of bone morphogenetic protein signaling in vivo regulates sys- temic iron balance. J. Clin. Invest. 117:1933-1939. doi:10.1172/JCI31342.

4. Wrighting, D.M., and Andrews, N.C. 2006. Interleukin-6 induces hepcidin expression through STAT3. Blood. 108:3204-3209.

5. Pietrangelo, A., et al. 2007. STAT3 is required for IL-6-gp130-dependent activation of hepcidin in vivo. Gastroenterology. 132:294-300.

6. Verga Falzacappa, M.V., et al. 2007. STAT3 mediates hepatic hepcidin expression and its inflammatory stimulation. Blood. 109:353-358.

7. Massague, J., Seoane, J., and Wotton, D. 2005. Smad transcription factors. Genes Dev. 19:2783-2810.

8. Wang, R.H., et al. 2005. A role of SMAD4 in iron metabolism through the positive regulation of hepcidin expression. Cell Metab. 2:399-409.

9. Babitt, J.L., et al. 2006. Bone morphogenetic protein signaling by hemojuvelin regulates hepcidin expression. Nat. Genet. 38:531-539.

10. Papanikolaou, G., et al. 2004. Mutations in HFE2 cause iron overload in chromosome 1q-linked juvenile hemochromatosis. Nat. Genet. 36:77-82.
11. Lin, L., Goldberg, Y.P., and Ganz, T. 2005. Competitive regulation of hepcidin mRNA by soluble and cell-associated hemojuvelin. Blood. 106:2884-2889.

12. Zhang, A.S., et al. 2007. Evidence that inhibition of hemojuvelin shedding in response to iron is mediated through neogenin. J. Biol. Chem. 282:12547-12556.

13. Fukuda, S., et al. 2007. Potentiation of astrogliogenesis by STAT3-mediated activation of BMPSmad signaling in neural stem cells. Mol. Cell. Biol. doi:10.1128/MCB.02435-06.

14. Truksa, J., Peng, H., Lee, P., and Beutler, E. 2006. Bone morphogenetic proteins 2, 4, and 9 stimulate murine hepcidin 1 expression independently of Hfe, transferrin receptor 2 (Tfr2), and IL-6. Proc. Natl. Acad. Sci. U. S. A. 103:10289-10293.

15. Goswami, T., and Andrews, N.C. 2006. Hereditary hemochromatosis protein, HFE, interaction with transferrin receptor 2 suggests a molecular mechanism for mammalian iron sensing. J. Biol. Chem. 281:28494-28498.

\section{Chain-reaction $\mathrm{Ca}^{2+}$ signaling in the heart}

\section{Sandor Györke,1 Brian M. Hagen,2 Dmitry Terentyev, ${ }^{1}$ and W. Jonathan Lederer²}

1Department of Physiology and Cell Biology and OSU Dorothy M. Davis Heart and Lung Research Institute, The Ohio State University, Columbus, Ohio, USA. 2Institute of Molecular Cardiology, Medical Biotechnology Center, University of Maryland Biotechnology Institute, Baltimore, Maryland, USA.

\begin{abstract}
Mutations in $\mathrm{Ca}^{2+}$-handling proteins in the heart have been linked to exercise-induced sudden cardiac death. The best characterized of these have been mutations in the cardiac $\mathrm{Ca}^{2+}$ release channel known as the ryanodine receptor type 2 (RyR2). RyR2 mutations cause "leaky" channels, resulting in diastolic $\mathrm{Ca}^{2+}$ leak from the sarcoplasmic reticulum (SR) that can trigger fatal cardiac arrhythmias during stress. In this issue of the JCI, Song et al. show that mutations in the $\mathrm{SR} \mathrm{Ca}^{2+}$-binding protein calsequestrin 2 (CASQ2) in mice result not only in reduced CASQ2 expression but also in a surprising, compensatory elevation in expression of both the $\mathrm{Ca}^{2+}$-binding protein calreticulin and RyR2, culminating in premature $\mathrm{Ca}^{2+}$ release from cardiac myocytes and stress-induced arrhythmia (see the related article beginning on page 1814). In the context of these findings and other recent reports studying CASQ2 mutations, we discuss how CASQ2 influences the properties of $\mathrm{Ca}^{2+}$-dependent regulation of RyR2 and how this contributes to cardiac arrhythmogenesis.
\end{abstract}

\section{Intracellular $\mathrm{Ca}^{2+}$ handling: \\ how the beat goes on}

In the normal heart, the cycling of intracellular $\mathrm{Ca}^{2+}$ in cardiomyocytes is critical to the heart's mechanical contraction and relaxation. On a beat-to-beat basis, $\mathrm{Ca}^{2+}$ entry through voltage-gated $\mathrm{Ca}^{2+}$

Nonstandard abbreviations used: $\left[\mathrm{Ca}^{2+}\right]_{\mathrm{i}}$, cytosolic $\mathrm{Ca}^{2+}$ concentration; $\left[\mathrm{Ca}^{2+}\right]_{\mathrm{SR}}$, SR Ca ${ }^{2+}$ concentration; CASQ2, calsequestrin 2; CICR, $\mathrm{Ca}^{2+}$-induced $\mathrm{Ca}^{2+}$ release; $\mathrm{CPVT}$, catecholamine-induced polymorphic ventricular tachycardia; jSR, junctional SR; PLN, phospholamban; $\mathrm{P}_{\mathrm{o}}$, open probability; RyR2, ryanodine receptor type 2; SERCA2a, sarcoplasmic or endoplasmic reticulum $\mathrm{Ca}^{2+}$ ATPase 2 ; SR, sarcoplasmic reticulum.

Conflict of interest: The authors have declared that no conflict of interest exists.

Citation for this article: J. Clin. Invest. 117:1758-1762 (2007). doi:10.1172/JCI32496. channels in the sarcolemma (including the transverse tubules) locally activates cardiac ryanodine receptor type 2 (RyR2) $\mathrm{Ca}^{2+}$ release channels found in large clusters in the junctional sarcoplasmic reticulum (jSR) across a very narrow (15 $\mathrm{nm})$ junctional gap (Figure 1A). The release of $\mathrm{Ca}^{2+}$ from functional clusters of RyR2s depends on this $\mathrm{Ca}^{2+}$ influx and works by the mechanism of $\mathrm{Ca}^{2+}$-induced $\mathrm{Ca}^{2+}$ release (CICR). The release of $\mathrm{Ca}^{2+}$ from the RyR2 clusters is visualized as $\mathrm{Ca}^{2+}$ sparks. The $\mathrm{Ca}^{2+}$ sparks amplify the initial $\mathrm{Ca}^{2+}$ influx trigger signal and combine to produce an elevation of cell-wide myoplasmic $\left[\mathrm{Ca}^{2+}\right]$ called the $\mathrm{Ca}^{2+}$ transient. This increase in cytosolic $\mathrm{Ca}^{2+}$ concentration $\left(\left[\mathrm{Ca}^{2+}\right]_{i}\right)$ leads to activation of the contractile proteins and hence to generation of the heartbeat (1). $\mathrm{Ca}^{2+}$ release to the cytosol is accompanied by a reciprocal decline in the $\left[\mathrm{Ca}^{2+}\right]$ within the SR $\left(\left[\mathrm{Ca}^{2+}\right]_{\mathrm{SR}}\right)$ for both the $\left[\mathrm{Ca}^{2+}\right]_{i}$ transient (2) and for $\mathrm{Ca}^{2+}$ sparks (3). This reduction in $\left[\mathrm{Ca}^{2+}\right]_{\mathrm{SR}}$ contributes to deactivation or closure of RyR2s, resulting in $\mathrm{Ca}^{2+}$ release termination and induction of a refractory state that prevents $\mathrm{Ca}^{2+}$ release during the diastole (4-6). Relaxation occurs following $\mathrm{Ca}^{2+}$ reuptake into the SR through the phospholamban-regulated (PLN-regulated) sarcoplasmic or endoplasmic reticulum $\mathrm{Ca}^{2+}$ ATPase 2 (SERCA2a). Ordered $\mathrm{Ca}^{2+}$ cycling is essential to normal rhythmic activity of the heart, and disturbances in $\mathrm{Ca}^{2+}$ handling have previously been shown to underlie diverse $\mathrm{Ca}^{2+}$-dependent cardiac arrhythmias (7-9). In this issue of the $J C I$, catecholamine-induced polymorphic ventricular tachycardia (CPVT) caused by mutations in calsequestrin 2 (CASQ2) is the subject of the study by Song et al. (10); $\mathrm{Ca}^{2+}$ signaling plays a central role in the dysfunction.

It is appreciated that RyR2 activity underlies $\mathrm{SR} \mathrm{Ca}^{2+}$ release and generation of the cytosolic $\mathrm{Ca}^{2+}$ transient that is required for muscle contraction. However, just how RyR2 is activated, how sensitive it is to $\left[\mathrm{Ca}^{2+}\right]$ in both cytosolic and lumenal compartments, and how RyR2-mediated $\mathrm{Ca}^{2+}$ release from the $\mathrm{SR}$ is regulated depends in 